\title{
EVALUATING UNIFORM MANIFOLD APPROXIMATION AND PROJECTION FOR DIMENSION REDUCTION AND VISUALIZATION OF POLINSAR FEATURES
}

\author{
Sylvia Schmitz ${ }^{1,2, *}$, Uwe Weidner ${ }^{2}$, Horst Hammer ${ }^{1}$, Antje Thiele ${ }^{1,2}$ \\ ${ }^{1}$ Fraunhofer IOSB, Ettlingen, Fraunhofer Institute of Optronics, System Technologies and Image Exploitation IOSB - \\ (Sylvia.Schmitz, Antje.Thiele, Horst.Hammer)@iosb.fraunhofer.de \\ ${ }^{2}$ Institute of Photogrammetry and Remote Sensing, Karlsruhe Institute of Technology (KIT) - \\ (Sylvia.Schmitz, Uwe.Weidner, Antje.Thiele)@kit.edu
}

Commission I, WG I/3

KEY WORDS: PolInSAR, F-SAR, Visualization, Dimension Reduction, UMAP.

\begin{abstract}
:
In this paper, the nonlinear dimension reduction algorithm Uniform Manifold Approximation and Projection (UMAP) is investigated to visualize information contained in high dimensional feature representations of Polarimetric Interferometric Synthetic Aperture Radar (PolInSAR) data. Based on polarimetric parameters, target decomposition methods and interferometric coherences a wide range of features is extracted that spans the high dimensional feature space. UMAP is applied to determine a representation of the data in 2D and 3D euclidean space, preserving local and global structures of the data and still suited for classification. The performance of UMAP in terms of generating expressive visualizations is evaluated on PolInSAR data acquired by the F-SAR sensor and compared to that of Principal Component Analysis (PCA), Laplacian Eigenmaps (LE) and t-distributed Stochastic Neighbor embedding (t-SNE). For this purpose, a visual analysis of $2 \mathrm{D}$ embeddings is performed. In addition, a quantitative analysis is provided for evaluating the preservation of information in low dimensional representations with respect to separability of different land cover classes. The results show that UMAP exceeds the capability of PCA and LE in these regards and is competitive with t-SNE.
\end{abstract}

\section{INTRODUCTION}

In recent years, Polarimetric Synthetic Aperture Radar (PolSAR) systems, which capture high resolution data independent of weather and daylight, have proven to be a powerful tool for earth observation. The information contained in fully polarimetric data can provide insights into the shape, orientation, and dielectric properties of observed scatterers on the ground. The high information content of the data enables applications ranging from land use and land cover classification, soil moisture and surface roughness estimation to ice thickness determination (Lee and Pottier, 2017, Cloude, 2009). To extract relevant information from polarimetric data, a number of specialized features have been introduced in recent years. These are either based directly on the backscattering coefficients, second order statistics represented by the covariance matrix or result from target decompositions, which aim at quantifying individual scattering mechanisms to facilitate a physical interpretation.

If a scene is covered from at least two slightly different angles or with a time offset, further valuable information can be obtained from the phase difference between the complex radar images. This imaging technique, which is referred to as Interferometric Synthetic Aperture Radar (InSAR), enables the derivation of geophysical parameters such as surface topography or ground deformation. The combination of polarimetry and interferometry, known as PolInSAR (Cloude and Papathanassiou, 1998), provides a rich source of information that allows the realization of a wide range of applications from biomass estimation to extended topography mapping.

\footnotetext{
* Corresponding author
}

Getting comprehensive and understandable overview of the vast amount of information that can be extracted from PolInSAR data is challenging. On the one hand, the visualization of different physically interpretative feature images is possible. For example, the components derived by a Freeman Durden decomposition encoded as a pseudocolor image is particularly suitable for the investigation of natural targets. On the other hand, different features aim at characterizing different properties of observed scatterers and it is not feasible to perceive all feature images separately to make a statement about the contained information. It is therefore desirable to generate a human interpretable visualization that summarizes the information content of a wide range of features in a comprehensive way.

In addition to the challenge of information gathering by human observers, a high number of features can likewise complicate automatic analysis. Especially in machine pattern recognition, as example for classification, regression or clustering of data, the complexity of estimators increases with an increasing number of feature dimensions or even leads to a loss of performance. This phenomenon is known as the curse of dimensionality.

To counteract this problem, a number of dimension reduction methods have been introduced. These methods aim at projecting high dimensional data into a space with fewer dimensions, retaining the important information to the maximum possible extent and removing redundant information. Among the most popular linear transformation methods are Principal Component Analysis (PCA) and Linear Discriminant Analysis (LDA). More recently presented approaches, which fall into the category of nonlinear dimension reduction, are mostly based on manifold learning techniques and aim in particular at obtaining the local structure of the data. These include, among others, Locally Linear Embeddings (LLE) (Roweis and 
Saul, 2000), Isometric Feature Mapping (ISOMAP) (Tenenbaum et al., 2000), Laplacian Eigenmaps (LE) (Belkin and Niyogi, 2003) and t-distributed Stochastic Neighbor embedding (t-SNE) (Van der Maaten and Hinton, 2008). Particularly for data visualization, $\mathrm{t}$-SNE is considered as a state-of-the-art approach.

In the context of PolSAR data, dimension reduction methods are typically used for preprocessing in classification processes. For example, in (Tu et al., 2012) LE are employed to represent the information from 42 different polarimetric features in only few components on the basis of which a Support Vector Machine (SVM) is applied for land cover classification. With a similar objective, the same high dimensional feature representation is used in (Shi et al., 2013a) and embedded into a low dimensional space using a supervised graph embedding. In (Ainsworth and Lee, 2004), the improvement of classification accuracy by preprocessing using ISOMAP is evaluated. More recently, manifold learning algorithms such as t-SNE and Locality Preserving Projections (LPP) are integrated into deep learning processes to combine principal features that reflect local back scattering properties with deep spatial features that capture a larger semantic context (He et al., 2020a, He et al., 2020b).

In 2018, McInnes et al. introduced a new manifold learning algorithm for general dimension reduction named UMAP (McInnes et al., 2018a), which is based on topological data analysis. It has been adopted for data analysis in several application domains, including mass spectrometry imaging (Smets et al., 2019), single cell data biology (Becht et al., 2019) and population genetics (Diaz-Papkovich et al., 2019). The evidence collected in the various studies suggests that UMAP has superior properties compared to other dimension reduction and visualization methods in terms of preservation of global data structure, transparency of parameters, computational speed and scalability towards large data sets.

To the best of our knowledge, UMAP has not yet been considered for the reduction of high dimensional features derived from PolInSAR data. Hence, this paper investigates the potential of UMAP for the visualization and explorative analysis of PolInSAR data. The objective is to assess the expressiveness of visualizations based on polarimetric and interferometric features embedded into 2D and 3D euclidean feature space using UMAP compared to other dimension reduction approaches. For this purpose, a visual analysis and comparison of $2 \mathrm{D}$ embeddings based on resulting scatter plots is carried out. Since this type of analysis is inherently subjective to a certain extent, an additional quantitative analysis is performed, which evaluates the preservation of information with regard to the separability of land cover classes. Further, we propose to visualize embedded features derived by UMAP within a spatial context by generating pseudo color images. These images can be used to easily identify the potential of available PolInSAR data, for example in terms of distinguishing different land cover classes or bio-physical properties.

\section{DATA SET}

The data that is explored in this paper was acquired by the F-SAR system during a measurement campaign in 2019. F-SAR is an airborne SAR system that enables the simultaneous acquisition of fully polarimetric data in multiple frequency bands (X-, C-, S-, L- and P-bands) (Horn et al., 2009).
Additionally, interferometric measurements can be realized in repeat-pass and single-pass. The data of the conducted measurement campaign are fully polarimetric S-band and VV polarized X-band data, recorded in single- and repeat-pass. Of the resulting available data products, only a subset is considered in this paper. This consists of the individual S-band Single Look Complex (SLC) images in each polarization and co-registered InSAR image pairs in VV polarization from single- and repeatpass measurements. However, since our visualization approach is easily expandable to a wider range of data, the other available products will also be included in the future.

\section{METHODS}

A wide variation of polarimetric and interferometric features is extracted from the selected data. While individual features can each characterize only a specific portion of the information contained in the data, the combination of partly complementary features provides a comprehensive description. Therefore, all extracted features are included to span a high dimensional feature space. Thus, each pixel in the SAR image is represented as a point in this space. In order to generate a low dimensional representation that can be visualized, the high dimensional feature representation is embedded into $2 \mathrm{D}$ and $3 \mathrm{D}$ euclidian space using UMAP. Resulting 2D embeddings are presented as scatter plots. Based on the 3D embeddings, pseudo color images are generated to allow observing the results within a spatial context.

\subsection{PolInSAR features}

To reduce the high level of speckle noise, the original SLC images are first down sampled by a factor of 3 and then filtered using the Refined Lee filter (Lee, 1981). Subsequently, several polarimetric and interferometric parameters and features, which are given in Table 1, are extracted using the PolSARpro software (Pottier et al., 2018). The first group of parameters contains the polarimentric backscattering coefficients and their ratios. Features based on polarimetric decompositions, as well as further polarimetric features are included in the second group. From the wide range of target decompositions, the Pauli decomposition, belonging to the category for the description of coherent scatterers, and the Van Zyl, Huynen and Cloude decomposition, which aim at characterizing distributed scatterers, are selected. Additionally, polarimetric properties described by the depolarization index, conformity coefficient, scattering predominance and the scattering diversity are included. For a detailed description of the calculation and interpretation of the different polarimetric features, please refer to (Lee and Pottier, 2017). In addition to descriptions of polarimetric properties, interferometric coherences are included, which quantify local phase correlations between two complex images.

The list of parameters and features given here does not represent a complete coverage of all available and potentially profitable features. However, for the intended evaluation of UMAP in the context of PolInSAR data representation, we limit ourselves to this selection. Nevertheless, modification and extension of features are quite conceivable.

In order to facilitate the validation and interpretation of results, all 27 feature images are projected into ground range under the flat earth assumption. The resulting images, with a resolution of $1 \mathrm{~m} \times 1 \mathrm{~m}$, are each normalized to the value range $[0,1]$ and then assembled into a 27-channel image array. Each point in the image is thus characterized by a 27 dimensional feature representation. 


\begin{tabular}{|c|c|}
\hline Expression & Physical Description \\
\hline$\sigma_{h h}^{0}=\left\langle S_{h h} S_{h h}^{*}\right\rangle$ & Co-polarized HH backscattering coefficient \\
\hline$\sigma_{h v}^{0}=\left\langle S_{h v} S_{h v}^{*}\right\rangle$ & Cross-polarized HV backscattering coefficient \\
\hline$\sigma_{v h}^{0}=\left\langle S_{v h} S_{v h}^{*}\right\rangle$ & Cross-polarized VH backscattering coefficient \\
\hline$\sigma_{v v}^{0}=\left\langle S_{v v} S_{v v}^{*}\right\rangle$ & Co-polarized VV backscattering coefficient \\
\hline$S P A N(\mathbf{S})=\left|S_{h h}\right|^{2}+\left|S_{h v}\right|^{2}+\left|S_{v h}\right|^{2}+\left|S_{v v}\right|^{2}$ & Total scattered power \\
\hline$r_{h h v v}=10 \log \left(\left|S_{h h}\right|^{2} /\left|S_{v v}\right|^{2}\right)$ & Co-polarized ratio in $\mathrm{dB}$ \\
\hline$r_{h v h h}=10 \log \left(\left|S_{h v}\right|^{2} /\left|S_{h h}\right|^{2}\right)$ & Cross-polarized ratio in $\mathrm{dB}$ \\
\hline$r_{h v v v}=10 \log \left(\left|S_{h v}\right|^{2} /\left|S_{v v}\right|^{2}\right)$ & Cross-polarized ratio in $\mathrm{dB}$ \\
\hline$|a|^{2},|b|^{2},|c|^{2}$ & $\begin{array}{l}\text { Contribution of scattered power of isotropic odd, even and } \pi / 4 \\
\text { even scatterers derived from Pauli decomposition (Cloude and } \\
\text { Pottier, 1996) }\end{array}$ \\
\hline$H, A, \alpha$ & $\begin{array}{l}\text { Entropy, anisotropy and alpha angle derived from Cloude decom- } \\
\text { position (Cloude and Pottier, 1997) }\end{array}$ \\
\hline$\underline{\lambda}=\sum_{i=1}^{3} p_{i} \lambda_{i}$ & $\begin{array}{l}\text { Weighted mean of eigenvalues of coherence matrix derived from } \\
\text { Cloude decomposition }\end{array}$ \\
\hline$A_{0}, B_{0}+B, B_{0}-B$ & $\begin{array}{l}\text { Symmetry, nonsymmetry and irregularity derived from Huynen } \\
\text { decomposition (Huynen, 1970) }\end{array}$ \\
\hline$f_{\text {odd }}, f_{\text {double }}, f_{\text {volume }}$ & $\begin{array}{l}\text { Odd-bounce, double-bounce and volume scattering derived from } \\
\text { Van Zyl decomposition (Van Zyl, 1993) }\end{array}$ \\
\hline$d(\mathbf{M})$ & Deplorization index (Gil and Bernabeu, 1985) \\
\hline$\mu$ & Conformity coefficient (Truong-Loi et al., 2009) \\
\hline$s(\mathbf{N})$ & Scattering predominance (Praks et al., 2009) \\
\hline$\hat{H}$ & Scattering diversity (Praks et al., 2009) \\
\hline$\left|\gamma_{v v, \text { single }}\right|=\left|\left\langle s_{v v, 1} s_{v v, 2}^{*}\right\rangle / \sqrt{\left\langle s_{v v, 1} s_{v v, 1}^{*}\right\rangle\left\langle s_{v v, 2} s_{v v, 2}^{*}\right\rangle}\right|$ & $\begin{array}{l}\text { Magnitude of interferometric coherence derived from single-pass } \\
\text { image pair }\end{array}$ \\
\hline$\left|\gamma_{v v, \text { repeat }}\right|=\left|\left\langle s_{v v, 1} s_{v v, 2}^{*}\right\rangle / \sqrt{\left\langle s_{v v, 1} s_{v v, 1}^{*}\right\rangle\left\langle s_{v v, 2} s_{v v, 2}^{*}\right\rangle}\right|$ & $\begin{array}{l}\text { Magnitude of interferometric coherence derived from repeat-pass } \\
\text { image pair }\end{array}$ \\
\hline
\end{tabular}

Table 1. Polarimetric and interferometric features, extracted to build a high dimensional representation of PolInSAR data.

\subsection{Uniform Manifold Approximation and Projection}

UMAP is applied to embed the high dimensional feature representation into a low dimensional euclidian space. At this point, only the basic idea behind the UMAP algorithm is presented. A detailed description of the underlying mathematical methods can be found in (McInnes et al., 2018a). UMAP belongs to the group of nonlinear dimension reduction methods and is based on manifold learning techniques. Under the assumption that high dimensional data are uniformly distributed on local manifolds, a topological representation of the data is constructed. This representation can basically be understood as a weighted graph in which edge weights indicate the probabilities of two data points being connected. To decide about the existence of an edge, open balls with a radius depending on the distance to the $n^{t} h$ nearest neighbor is drawn outward from each point. Subsequently, the layout of an equivalent representation in the low dimensional space is constructed and optimized to resemble the topological structure of the high dimensional representation as closely as possible. This is realized by minimizing the crossentropy between the two topological representations.

\subsection{Data visualization}

Dimension reduction algorithms are valuable to generate human interpretable visualizations that help to understand the underlying structure of available data. One way to visualize the PolInSAR feature representation reduced to 2 and 3 dimensions is by using scatter plots, where each pixel is represented as a data point in $x-y$ plane and $x-y-z$ space, respectively. The relative position of the points to each other represents the similarity of back scattering properties encoded in PolInSAR features. Scatter plots of $2 \mathrm{D}$ embeddings generated using UMAP are presented and visually compared to scatter plot of $2 \mathrm{D}$ embeddings generated with comparable dimension reduction methods in Section 4.1. A quantitative evaluation with regard to the informative value contained within the different embeddings is presented in Section 4.2.

Scatter plots can be useful to detect clusters in the data and gain a deeper understanding of the relationship and internal structure of the data. However, spatial information is not captured in this type of visualization. To overcome this challenge, pseudo color images are derived from 3D embeddings. The $\mathrm{x}-, \mathrm{y}-$ and $\mathrm{z}$-components are scaled linearly to a range of values from 0 to 255 and interpreted as red, green and blue intensities. Visualizations generated in this manner are shown and discussed in Section 4.3.

\section{EXPERIMENTS}

The main objective of our research is to assess the information content and quality of visualizations derived from PolInSAR features embedded into 2D and 3D euclidean space. To evaluate in particular the preservation of local structures of original 

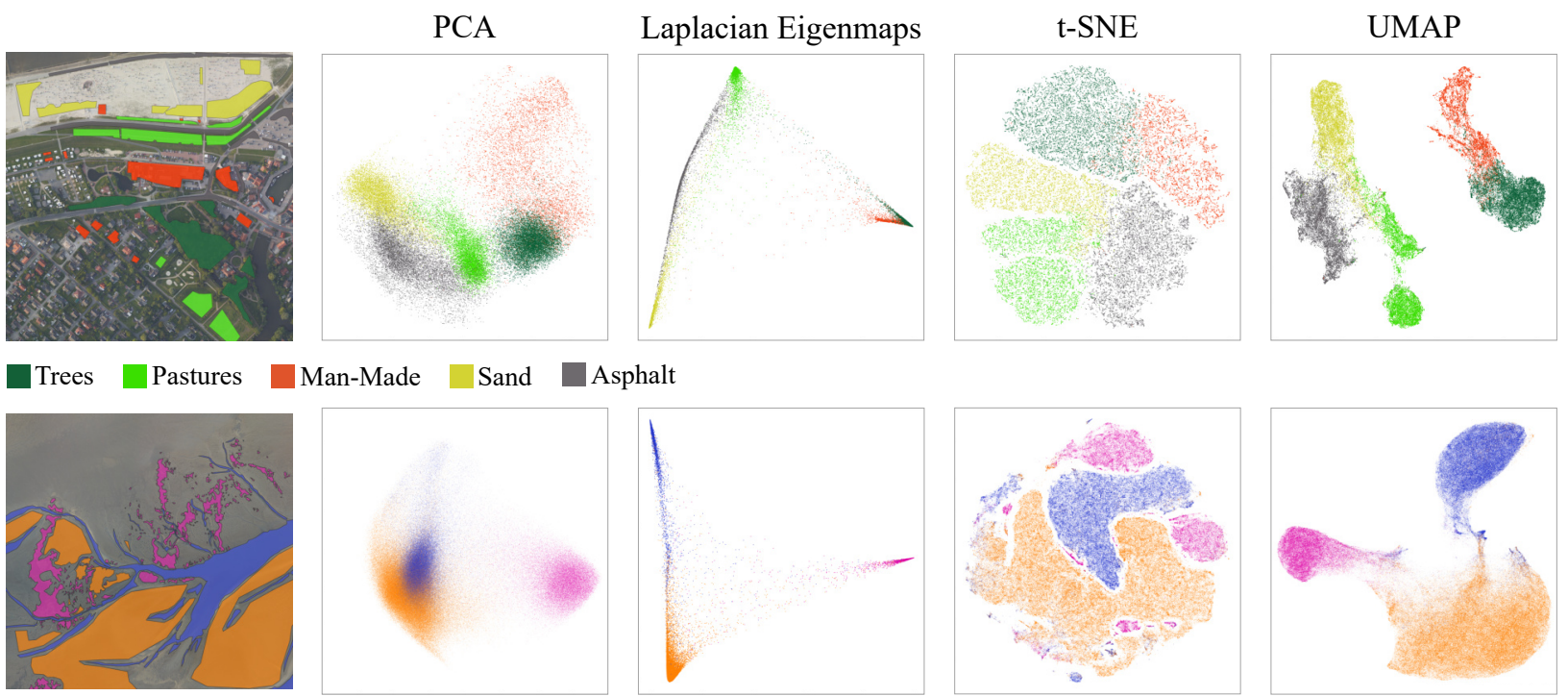

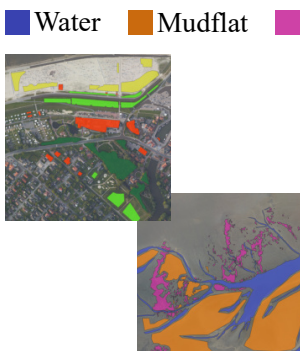

(a)

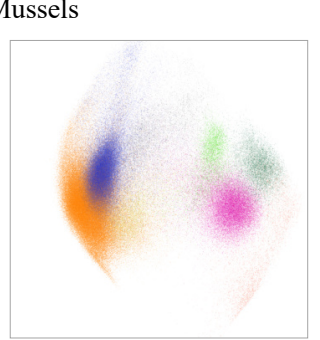

(b)

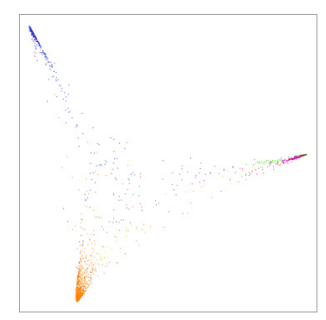

(c)

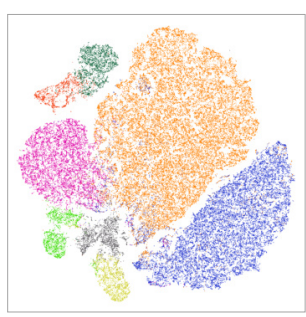

(d)

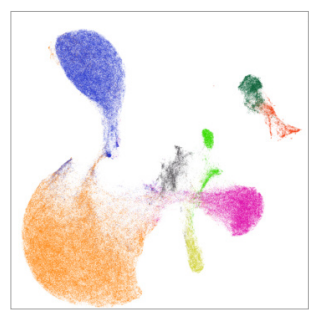

(e)

Figure 1. Comparison of 2D feature representations, derived by different dimension reduction methods. In (a) orthophotos of the selected scenes are given, overlaid with labeled reference classes. In (b) to (e) $2 \mathrm{D}$ embeddings derived from PCA, LE, t-SNE and

UMAP are shown. Embeddings illustrated in the first and second row contain points from only one scene, while embeddings illustrated in the third row are joined embeddings calculated based on points of both scenes. Embedded data points are colored by their corresponding class label.

high dimensional data in the low dimensional representation, class information is included. The classes represent different land cover types, since it is expected that different land covers will differ in their backscattering properties reflected in the high dimensional feature representation.

UMAP is compared with other dimension reduction methods, including PCA, LE and t-SNE. PCA is probably one of the most commonly used methods for dimension reduction. Thereby, the high dimensional feature representation is approximated by a lower dimensional representation that captures most of the variance of the data. The LE method is a nonlinear, graph based approach in which particularly local information is preserved in the low dimensional embedding. LE is used for comparison in this work, as its successful application to PolSAR data was already demonstrated (Tu et al., 2012, Shi et al., 2013b). t-SNE is a further nonlinear dimension reduction algorithm based on neighborhood graphs that aims at preserving the local structure of data. The basic idea in this approach is, to convert distances between data points into probability distributions that should be as similar as possible to each other in high and low dimensional representations. Since t-SNE is regarded as state-of-theart method for the visualization of high dimensional data, it is included in the comparison.

For the comparison of the different dimension reduction methods, the implementations from the sci-kit learn software package (Pedregosa et al., 2011) for PCA, LE and the Barnes-Hut approximation of t-SNE are used with provided default parameters. For the implementation of UMAP, the UMAP python package (McInnes et al., 2018b) is used.

The experiments performed consist of three principal parts. First, a visual analysis of 2D embeddings is carried out based on resulting scatter plots. Secondly, in order to evaluate the preservation of information content with respect to class separation more objectively, a quantitative analysis based on supervised classification is performed. Thirdly, pseudo color images based on UMAP embeddings are explored.

\subsection{Visual analysis}

For the comparison of embeddings derived from different dimension reduction approaches, two exemplary SAR image sections that capture different land cover classes are selected. The first image section depicts an onshore coastal area and maps the classes trees, pastures, man-made objects, sand and asphalt. In the following, this section is referred to as onshore scene. In the second image section, denoted offshore scene, a tidal area is captured that is composed of water, tidal flats and mussels. Within the two scenes, reference areas representing the specified classes were manually marked with the help of optical aerial imagery (see Figure 1(a)). All data points, described by the high dimensional feature representation, located within the referenced areas are embedded into 2D euclidean space using the different dimension reduction algorithms. The resulting 


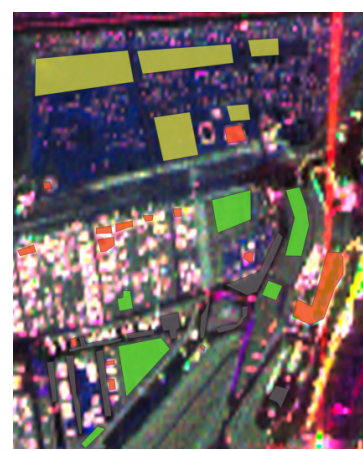

Pastures Man-Made

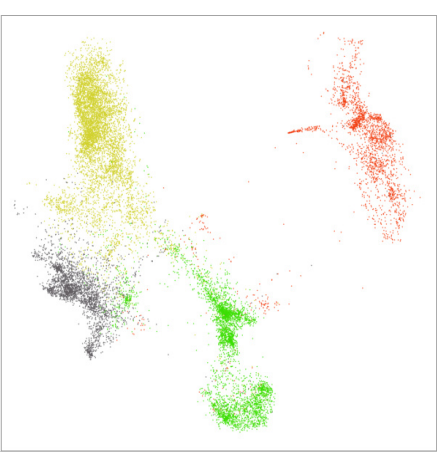

Sand

Asphalt

Figure 2. Embedding unknown test data in previously learned feature space. On the left the Pauli decomposition of the test scene overlaid with reference polygons is shown. On the right, the $2 \mathrm{D}$ embedding of test data is presented.

embeddings are presented as scatter plots in Figure 1(b) to (e), where data points are colored according to their reference class. First, the two scenes are analyzed separately (first two rows). Additionally, data points from both scenes are embedded jointly (last row). Regarding the PCA embedding, the following observations are derived from Figure 1: For both scenes, a spatial sorting by land cover class is clearly evident. However, the isolation of distinct clusters is only partially successful. The classes sand, asphalt and pastures transition into each other (onshore scene) and overlaps occur particularly between the water and tidal flat classes (offshore scene). Consistent with the underlying methodology, PCA embeddings primarily provide global information content and are unable to represent rich local data structures. The LE algorithm, which aims at preserving local data structure, provides a locally sparse but globally smooth representation in triangular shape. Considering both scenes independently, the separation of associated land classes is apparent. In the joint representation, however, the problem arises that the land cover classes represented by a smaller proportion of data points (trees, pastures, man-made objects, sand, asphalt and mussels) are projected into a dense space and are no longer visually separable. In contrast, t-SNE tends to distribute the data uniformly around the reduced feature space and achieves a clear separation of distinct clusters that correspond to different land cover classes. However, classes are not necessarily represented in only a single cluster. Particularly for the offshore scene, it can be observed that classes decompose into isolated non-adjacent subclusters. The 2D embeddings obtained by UMAP show comparable class separation ability to that of t-SNE embeddings. A striking difference is the denser packing of data points within individual clusters as well as the varying distances between clusters. A possible explanation for the different behavior regarding cluster distances is that t-SNE preserves only local data structure, while UMAP additionally aims at preserving global data structure. Methodologically, this is related to the choice of different cost functions used for the optimization as argued in (McInnes et al., 2018a).

An advantage of the UMAP method over t-SNE and LE is that the low dimensional feature representation learned on one data set is transferable to unknown test data. Thus, the approach allows adding new data points to an existing 2D visualization without computing a new feature space on all data. Further, higher flexibility is given in terms of its usability for machine learning tasks such as classifications. To investigate this functionality on the PolInSAR data, a test scene with similar land

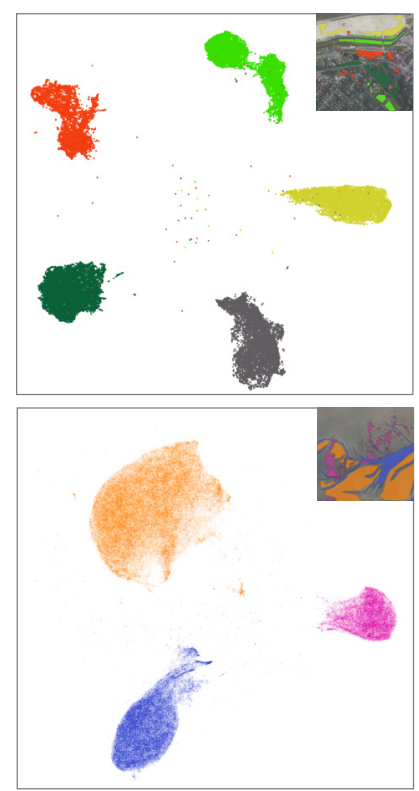

(a)

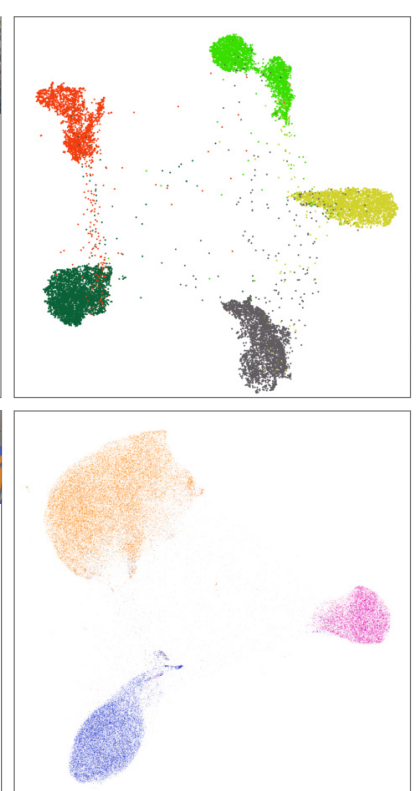

(b)
Figure 3. Supervised UMAP. The 2D embedding of the training data (a) and the (b) test data that are embedded in the learned 2D feature space (b) are illustrated.

cover to the onshore scene is involved. The unknown test data are embedded into the feature space that is learned on the data of the onshore scene. The result is shown in Figure 2. It is observed that data points are embedded in the expected locations in the 2D feature space. Also the internal shape and structure of clusters can be reproduced by embedding the unknown test data.

In addition to unsupervised dimension reduction, UMAP can also be used for supervised dimension reduction, in which the information about the class membership of a data point is used to determine an optimal embedding. The goal is to map different classes in the low dimensional space as far apart as possible, while at the same time maintaining the internal class structure and the inter-class relationships. To test the supervised variant of UMAP on the PolInSAR data, the data points of the onshore and offshore scene are divided into training data $(20 \%$ of the points) and test data ( $80 \%$ of the points). To learn an appropriate 2D embedding, only the training data with given class labels are used. Subsequently, the test data are embedded on this basis. Figure 3 illustrates the resulting scatter plots for training and test data. For the training data, a very clear class separation is realized, especially compared to the unsupervised version of UMAP. This was expected, since the information of the class membership was known for the determination of the embedding. Analysis of embedded test data, which was not included in the learning process, shows that the clear class separation is preserved. Only for a few data points a clear assignment to a cluster fails. In addition to the good class separation, it is evident that the internal structures of the individual classes are highly similar to those of unsupervised embedding. This indicates that structural properties of the data are retained, while applying supervised UMAP.

\subsection{Quantitative analysis}

In the following, we quantitatively analyze to what extent the different dimension reduction methods allow to preserve information of high dimensional PolInSAR features represented 


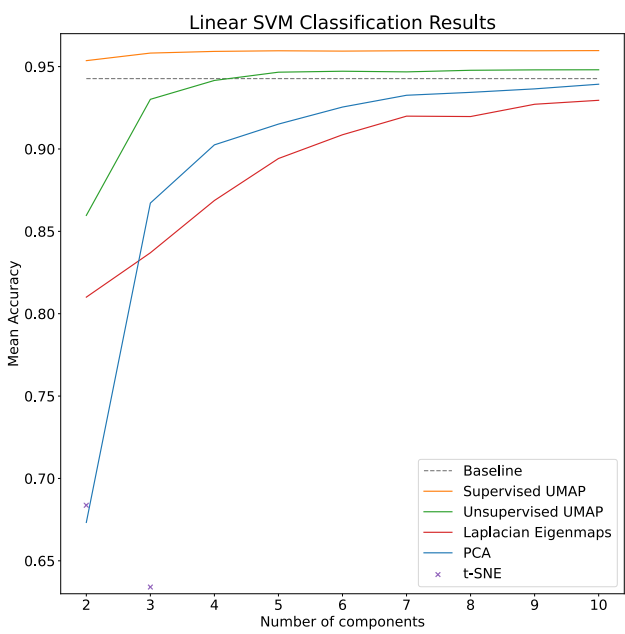

Figure 4. Mean accuracy for the SVM classification of embedded test data via UMAP, PCA, LE and t-SNE. Results are shown over increasing dimensions of embeddings.

within a minimal number of components. For this purpose, the linear separability of different land cover classes based on embeddings of increasing dimensions is considered. As a measure of quality, the classification accuracy achieved by applying a linear SVM is used. Notably, this analysis does not aim at determining the performance of the classifier, but at quantifying the preservation of local structures and linear separability in low dimensional representations.

All eight land cover classes present in the onshore and offshore test scenes are included in the classification. First, all labeled data points are divided into equally sized training and test sets. For dimension reduction performed by PCA and UMAP, only the training data are used to determine suitable embeddings and the test data are embedded into the learned space only afterwards. In contrast, dimension reduction performed by LE and $\mathrm{t}-\mathrm{SNE}$ is based on all data points (training and test set), since a transformation of non-included data is not possible. Based on embedded data points and their corresponding class labels linear SVM classifiers are trained for each embedding. Optimal penalty parameters are determined for each of the classifiers by applying a grid search with 5-fold cross validation based on the training set. Resulting classifiers are used to predict class labels for embedded held out test data points. Figure 4 presents the classification accuracy in dependence of embedding's dimension for each dimension reduction method. Since t-SNE only allows to embed data in two or three dimensions, only these two results are available. In addition, the accuracy labeled as baseline is provided, which presents the result of a linear SVM classification based on the original 27-dimensional feature representation.

Classifications based on supervised UMAP embeddings achieve highest accuracies across all dimensions. With accuracies of approximately $96 \%$, it provides better results even on the basis of the 2D representation than achievable using the high dimensional feature representation. The superiority of the supervised UMAP method was presumable, since it presents the only method in the comparison, in which the information of the class membership is included for the determination of a suitable embedding. However, the 2D and 3D embeddings obtained by unsupervised UMAP preserve the linear separability of the data compared to PCA, LE and t-SNE to a high degree as well. To

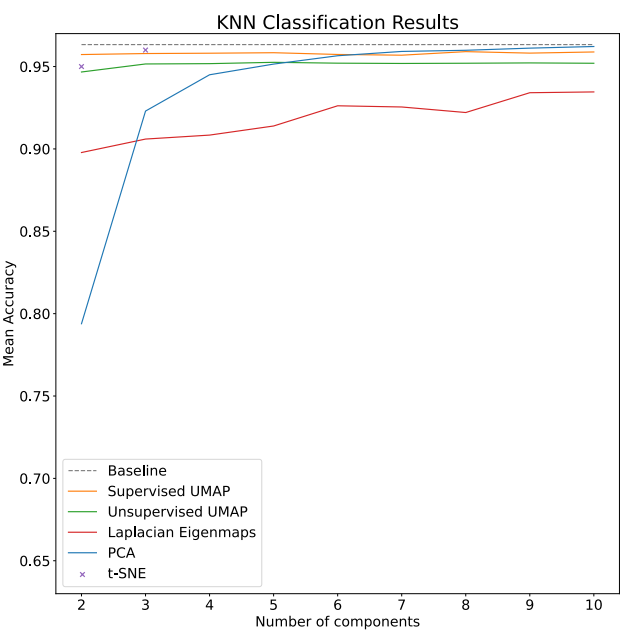

Figure 5. Mean accuracy for the KNN classification of embedded test data via UMAP, PCA, LE and t-SNE. Results are shown over increasing dimensions of embeddings.

outperform the classification performance based on high dimensional representation, only five dimensions are needed when using unsupervised UMAP. The poorer linear separability in the representations provided by t-SNE have already become apparent in the visual analysis, in which the nonlinear mapping was observable.

Another measure of quality, commonly used to evaluate dimension reduction and visualization methods, is the class separability by means of a k-nearest neighbors (KNN) classifier. The choice behind this benchmarking method is motivated by the fact that labels tend to be similar in small neighborhoods and therefore the classification accuracy based on neighborhoods would remain close to that in high dimensional space. If the dimension reduction method cannot maintain neighborhoods, a degradation of KNN accuracy is expected. For KNN accuracy based evaluation, the previously described process of parameter tuning (in this case the number of nearest neighbors), training and testing of classifiers is followed. Results are presented in Figure 5.

Especially for very low dimensions, the supervised UMAP method is again superior. In contrast to the SVM results, the $\mathrm{KNN}$ accuracies based on the 2D and 3D embeddings of t-SNE and unsupervised UMAP are comparable and with approximately $95 \%$ at a high level. It can be concluded that although linear separability is no longer present to the same extent as in higher dimensions, the local neighborhood structure is well preserved in very low dimensional representations.

\subsection{Data visualization results}

In the previous sections, it was shown that UMAP is suitable to represent a high amount of information contained in PolInSAR data in just a few components. To visualize this information within a spatial context, pseudo color images are generated using the method described in Section 3.3. In this representation, the coloring of each pixel reflects the location of the corresponding data point in the reduced 3D feature space. In Figure 6 (a) and (b), resulting pseudo color images are shown alongside the corresponding orthophotos of the onshore and offshore scene. The underlying 3D embeddings are learned on a subsets of the data points in an unsupervised manner. Remaining points were embedded accordingly. The reduced 3D feature 

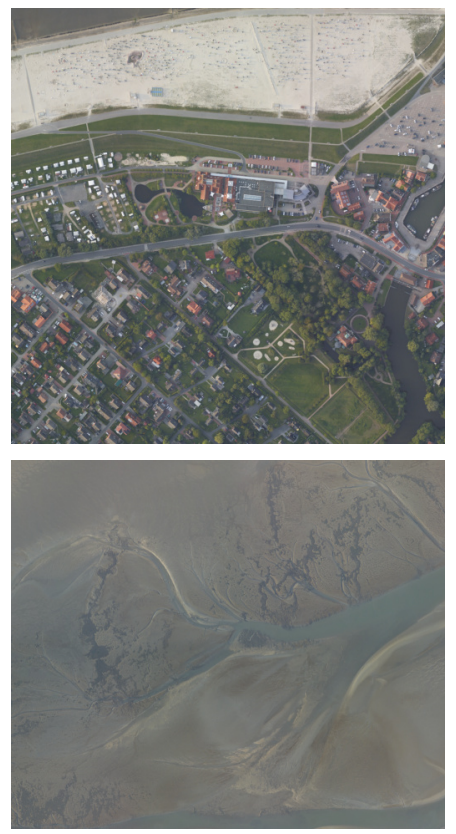

(a)
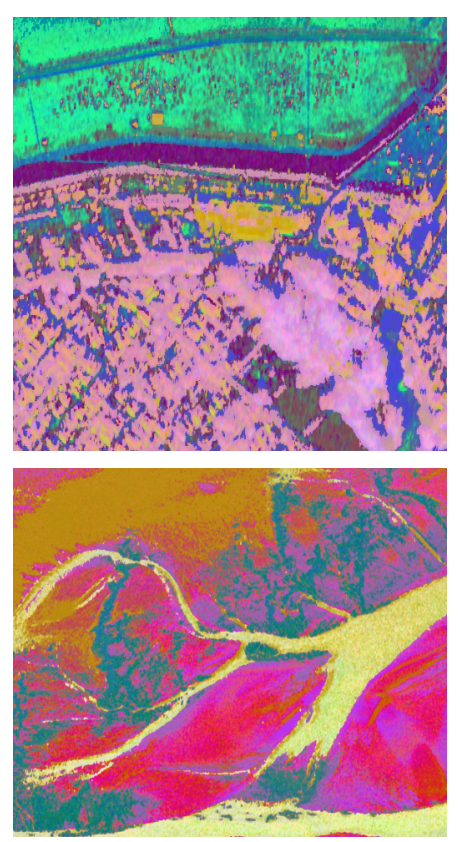

(b)
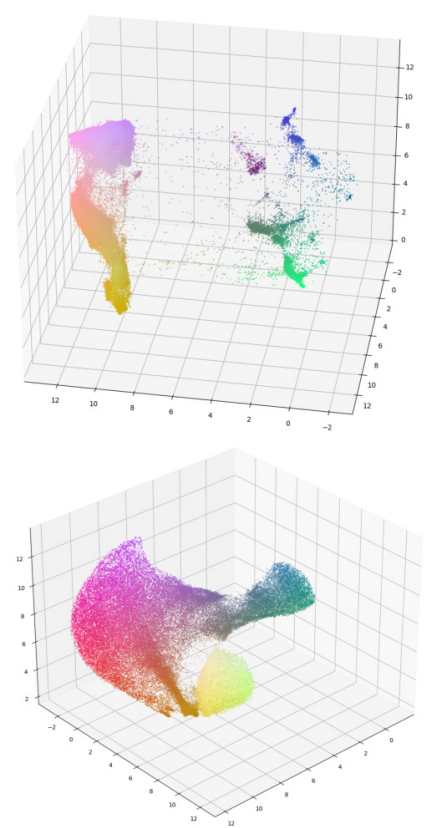

(c)

Figure 6. In (a) orthophotos of the onshore and offshore scene are given. (b) presents pseudo color images, which encode the 3D feature representation of the PolInSAR data. In (c) embedded data points are shown in 3D euclidean space.
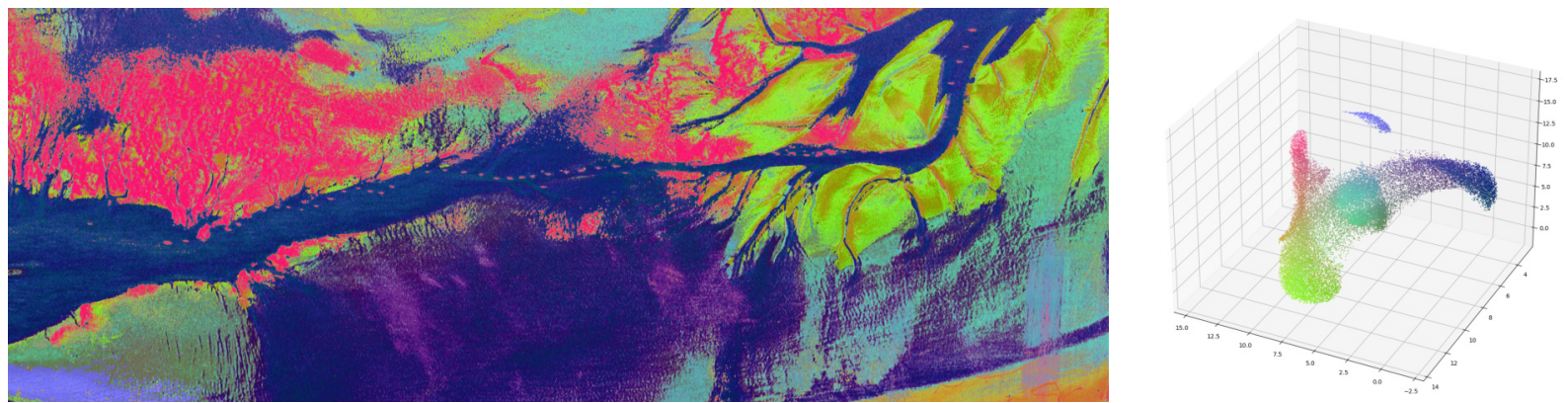

Figure 7. Pseudo color image of tidal area, covering $6,3 \mathrm{~km}^{2}$, and the corresponding 3D scatter plot.

spaces, containing all embedded data points are illustrated in Figure 6 (c). When comparing the pseudo color images to the optical images, a clear correlation between coloring and land cover is apparent. Especially for the offshore scene, the strong color contrasts enable land classes to be visually distinguished even better than in the optical image. Thus, without providing explicit class labels, pseudo color images can be used to intuitively identify which land cover classes are identifiable based on the PolInSAR data and which may be potentially more challenging to separate. A further observation concerns the transition regions between different land cover classes. In the pseudo color image of the offshore scene, dark green colored mussel beds are surrounded by dark purple tones that then transition to a pink color, which apparently represents the tidal flat class. The exact same color transition is also identifiable in the 3D scatter plot in the transition between two distinct clusters. It is reasonable to assume that data points located in such transition areas in the scatter plot either represent resolution cells in which two different land covers are present or areas that cannot be clearly assigned to a particular land cover class.

Figure 7, shows a resulting pseudo color image for a wider area. The color scheme with respect to the various land cover classes is inherently consistent throughout the scene. Mussels appear in pink, water in dark blue, and tidal flats are divided into green, light blue, and orange areas. The color differentiation between various tidal flat areas suggest that sediment types with different properties, such as water content and surface roughness, are distinguishable using PolInSAR data.

\section{CONCLUSION}

In this paper, we evaluated the potential of the nonlinear dimension reduction method UMAP for visualizing high dimensional feature representations of PolInSAR data. Thereby, this work represents the first approach to the use of UMAP in the context of PolInSAR data. The preservation of information content in low dimensions was considered with respect to the separability of different land cover classes. It is demonstrated that compared to commonly used methods like PCA, LE or t-SNE, the local and global structure of high dimensional PolInSAR feature representation is preserved superiorly. We conclude that UMAP provides a suitable framework to gain a deeper understanding of high dimensional feature representations of PolInSAR data. In particular, the generation of pseudo color images based on 3D embeddings is useful to visualize the information density of PolInSAR data in a human interpretable way. Furthermore, in the context of automated land cover classification, it is shown 
that low dimensional representations determined by UMAP are sufficient to achieve at least the same accuracies as the original high dimensional representation using simple classifiers. This suggests that in addition to the visualization being focused in this paper, UMAP can act as a useful building block in the development of classification processes.

\section{REFERENCES}

Ainsworth, T. L., Lee, J., 2004. Polarimetric SAR image classification-exploiting optimal variables derived from multiple-image datasets. IGARSS 2004. 2004 IEEE International Geoscience and Remote Sensing Symposium, 1, 188191.

Becht, E., McInnes, L., Healy, J., Dutertre, C.-A., Kwok, I. W., Ng, L. G., Ginhoux, F., Newell, E. W., 2019. Dimensionality reduction for visualizing single-cell data using UMAP. Nature Biotechnology, 37(1), 38-44.

Belkin, M., Niyogi, P., 2003. Laplacian Eigenmaps for Dimensionality Reduction and Data Representation. Neural Computation, 15(6), 1373-1396.

Cloude, S., 2009. Polarisation: Applications in Remote Sensing. OUP Oxford.

Cloude, S. R., Papathanassiou, K. P., 1998. Polarimetric SAR interferometry. IEEE Transactions on Geoscience and Remote Sensing, 36(5), 1551-1565.

Cloude, S. R., Pottier, E., 1996. A review of target decomposition theorems in radar polarimetry. IEEE Transactions on Geoscience and Remote Sensing, 34(2), 498-518.

Cloude, S. R., Pottier, E., 1997. An entropy based classification scheme for land applications of polarimetric SAR. IEEE Transactions on Geoscience and Remote Sensing, 35(1), 68-78.

Diaz-Papkovich, A., Anderson-Trocmé, L., Gravel, S., 2019. UMAP reveals cryptic population structure and phenotype heterogeneity in large genomic cohorts. PLoS Genetics, 15(11), $1-24$.

Gil, J. J., Bernabeu, E., 1985. A depolarization criterion in Mueller matrices. Optica Acta: International Journal of Optics, 32(3), 259-261.

He, C., He, B., Tu, M., Wang, Y., Qu, T., Wang, D., Liao, M., 2020a. Fully Convolutional Networks and a Manifold Graph Embedding-Based Algorithm for PolSAR Image Classification. Remote Sensing, 12(9), 1467-1489.

He, C., Tu, M., Xiong, D., Liao, M., 2020b. Nonlinear Manifold Learning Integrated with Fully Convolutional Networks for PolSAR Image Classification. Remote Sensing, 12(4), 655-677.

Horn, R., Nottensteiner, A., Reigber, A., Fischer, J., Scheiber, R., 2009. F-SAR-DLR's new multifrequency polarimetric airborne SAR. 2009 IEEE International Geoscience and Remote Sensing Symposium, 2, II:902-II:905.

Huynen, J. R., 1970. Phenomenological theory of radar targets. $\mathrm{PhD}$ thesis, Technical University Delft.

Lee, J.-S., 1981. Refined filtering of image noise using local statistics. Computer Graphics and Image Processing, 15(4), 380-389.
Lee, J.-S., Pottier, E., 2017. Polarimetric Radar Imaging: From Basics to Applications. CRC press.

McInnes, L., Healy, J., Melville, J., 2018a. Umap: Uniform manifold approximation and projection for dimension reduction. arXiv preprint arXiv:1802.03426.

McInnes, L., Healy, J., Saul, N., Grossberger, L., 2018b. UMAP: Uniform Manifold Approximation and Projection. The Journal of Open Source Software, 3(29), 861-862.

Pedregosa, F., Varoquaux, G., Gramfort, A., Michel, V., Thirion, B., Grisel, O., Blondel, M., Prettenhofer, P., Weiss, R., Dubourg, V., Vanderplas, J., Passos, A., Cournapeau, D., Brucher, M., Perrot, M., Duchesnay, E., 2011. Scikit-learn: Machine Learning in Python. Journal of Machine Learning Research, 12(85), 2825-2830.

Pottier, E., Ferro-Famil, L., Fitrzyk, M., Desnos, Y.-L., 2018. Polsarpro-bio: The new scientific toolbox for esa \& third party fully polarimetric sar missions. EUSAR 2018; 12th European Conference on Synthetic Aperture Radar, VDE, 1-4.

Praks, J., Koeniguer, E. C., Hallikainen, M. T., 2009. Alternatives to target entropy and alpha angle in SAR polarimetry. IEEE Transactions on Geoscience and Remote Sensing, 47(7), 2262-2274.

Roweis, S. T., Saul, L. K., 2000. Nonlinear dimensionality reduction by locally linear embedding. Science, 290(5500), 2323-2326.

Shi, L., Zhang, L., Yang, J., Zhang, L., Li, P., 2013a. Supervised Graph Embedding for Polarimetric SAR Image Classification. IEEE Geoscience and Remote Sensing Letters, 10(2), 216-220.

Shi, L., Zhang, L., Zhao, L., Yang, J., Li, P., Zhang, L., 2013b. The potential of linear discriminative Laplacian eigenmaps dimensionality reduction in polarimetric SAR classification for agricultural areas. ISPRS Journal of Photogrammetry and Remote Sensing, 86, 124-135.

Smets, T., Verbeeck, N., Claesen, M., Asperger, A., Griffioen, G., Tousseyn, T., Waelput, W., Waelkens, E., De Moor, B., 2019. Evaluation of distance metrics and spatial autocorrelation in uniform manifold approximation and projection applied to mass spectrometry imaging data. Analytical Chemistry, 91(9), 5706-5714.

Tenenbaum, J. B., De Silva, V., Langford, J. C., 2000. A global geometric framework for nonlinear dimensionality reduction. Science, 290(5500), 2319-2323.

Truong-Loi, M.-L., Dubois-Fernandez, P., Freeman, A., Pottier, E., 2009. The conformity coefficient or how to explore the scattering behaviour from compact polarimetry mode. 2009 IEEE Radar Conference, IEEE, 1-6.

Tu, S. T., Chen, J. Y., Yang, W., Sun, H., 2012. Laplacian Eigenmaps-Based Polarimetric Dimensionality Reduction for SAR Image Classification. IEEE Transactions on Geoscience and Remote Sensing, 50(1), 170-179.

Van der Maaten, L., Hinton, G., 2008. Visualizing data using t-SNE. Journal of Machine Learning Research, 9(11), 25792605.

Van Zyl, J. J., 1993. Application of Cloude's target decomposition theorem to polarimetric imaging radar data. Radar Polarimetry, 1748, 184-191. 